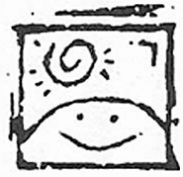

http://doi.org/10.15359/ree.2004-6.9

Educación Inicial

\title{
SEXISMO EN EL AULA PREESCOLAR COSTARRICENSE: UN ACERCAMIENTO A LA PERSPECTIVA DE NIÑOS Y NIÑAS
}
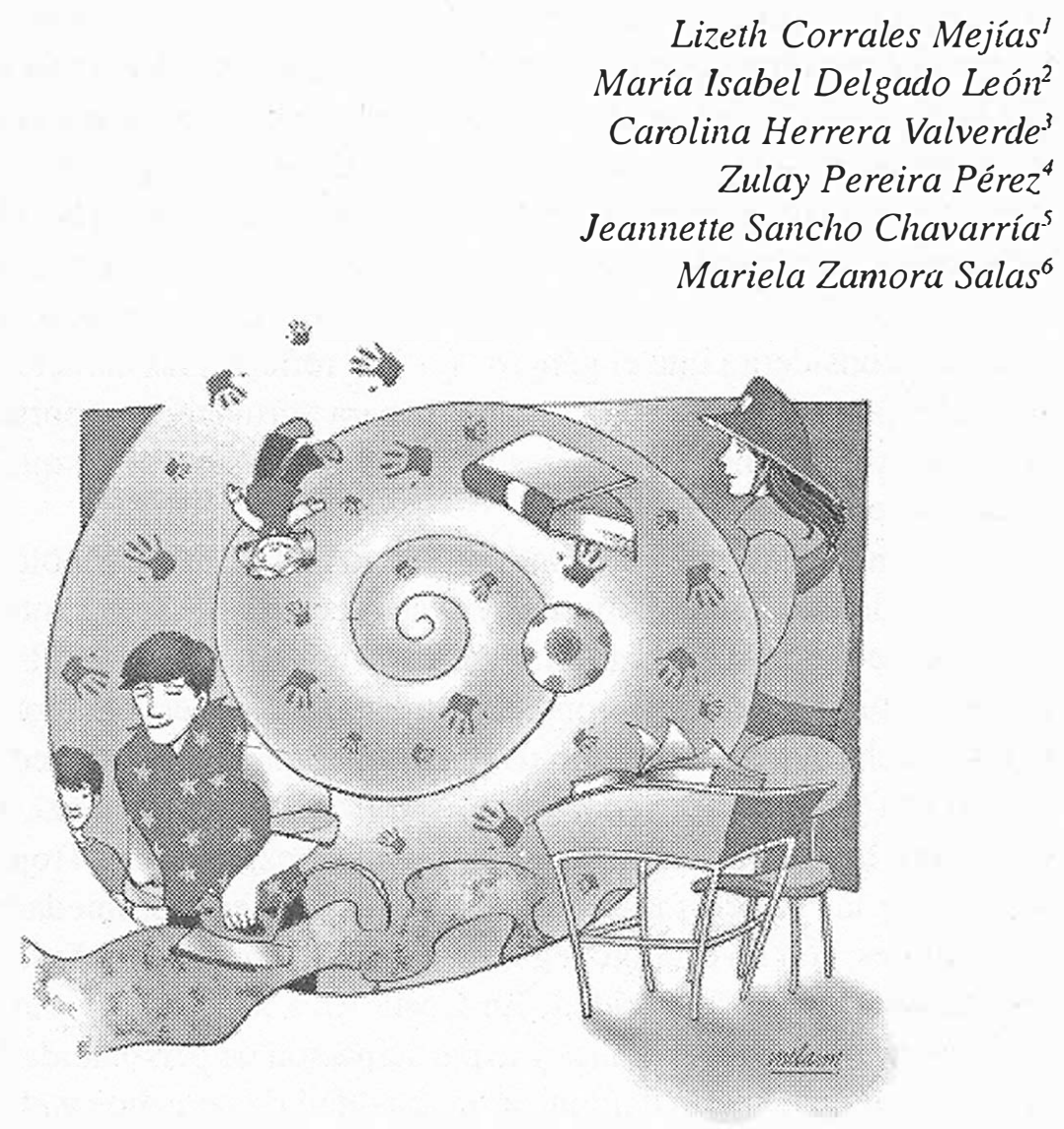

1 Licenciada en Educación Preescolar. Docente de Preescolar.

2 Licenciada en Educación Preescolar. Docente de Preescolar.

3 Licenciada en Educación Preescolar. Docente de Preescolar.

4 Catedrática de la Universidad Nacional. Máster en Psicología de la Universidad de Costa Rica.

5 Licenciada en Educación Preescolar. Docente de Preescolar.

6 Licenciada en Educación Preescolar. Docente de Preescolar. 

los resultados y conclusiones acerca de una

Cabe señalar que la condición de hombre o mujer no está determinada únicamente por el sexo de cada uno, para Ayales, Benítez, Fevrier y Ramírez (s.f.) no es solamente el "(...) aspecto biológico que tiene su expresión en las diferencias genitales y reproductivas" (p. 14), sino que va más allá tomando como referentes aspectos culturales y sociales que comúninvestigación realizada en instiruciones pre. escolares costarricenses acerca de la pers. pectiva del sexismo. En este primer arriculo se abordan las concepciones aporradas por los niños y las niñas parricipantes. En el sesundo articulo se retoman las opiniones de docentes de preescolar acerca de esta temárica. Se invita al lector a acercarse a la lectura de este primer articulo que se refiere a la perspectiva de niños y niñas costarricenses en relación con el sexismo.

mente se denominan como género. La palabra género denota toda una ideología que varía según la formación de cada ser humano. Cabe destacar que socialmente existe un lenguaje común para definir dicho concepto, responde así a una diferenciación de roles entre los sexos en donde se otorga al hombre y a la mujer características específicas que los distinguen y condicionan al mismo tiempo, las cuales suelen estar cargadas de estereotipos. De este modo los autores mencionados consideran que el género "(...) se refiere a las características sociales definidas por una sociedad determinada para normar el comportamiento, aptitudes, roles y funciones de mujeres y hombres y la manera en que deben relacionarse e interactuar" (p. 14).

El género hay que entenderlo como algo social y no biologico, ya que su génesis radica en bases culturales ya establecidas. Las constantes interacciones sociales mediante instancias tales como la familia, la religión, la educación, y la misma cultura provocan un condicionamiento que genera comportamientos aceptados o rechazados en el modo de vida imperante en una sociedad.

Hasta hace muy poco tiempo Aguilar, Barley, Bertozzi, Calvo, Camac, Camacho, Escalante, Facio, González. Gutiérrez, Quirós y Trejos, (1995) planleaban que las mujeres estuvieron reducidas a meras propiedades u objetos de los hombres, con un estatuto legal similar al de un niño o niña, un (a) demente o una persona con discapacidad. En Costa Rica a partir del año 1970, se da el surgimiento de organizaciones y la participación de personalidades con el deseo de erradicar el sexismo e impulsar la igualdad de derechos y el desarrollo integral de las mujeres. Como consecuencia de lo anterior, se crearon algunas instituciones entre las que pueden destacarse el Centro Nacional para el Desarrollo de la Mujer y la Familia, la Defensoria de la Mujer, asimismo se aprobó la Ley de Promoción Social de la Mujer. 
The present article discusses some of the resulis and conchusions wirhetrawn from a research carsicd oun in Cosia Rican preschools on the issue of sex discrimination. This first pars addie'sses the conceptions provided by the parricipaling boys and girls. The second part deats will slie opinions given by preschool teachers. We nams so imvite you, the reader, 10 exumine athis first article on she perspectiv's shared by Costu Rican boy's and girls on alie ropic of sexism.
Es así como, con el tiempo por razones culturales, económicas y políticas, se ha ido determinando el proceso de reconocimiento de la mujer en cuanto a su calidad de ser humano y' de ciudadana. Sin embargo, esta panorámica aún tiene un largo camino por recorrer.

En Costa Rica, según el Ministcrio de Educación Públiciı (MEP 2000-2002) existe la Ley de Promoción de la Igualdad Social de la Mujer (creada por la Asamblea Legislativa en 1990), la cual establece en el Capítulo $V$, artículo 17, la prohibición de que en cualquier institución educativa nacional, se utilicen comtenidos, métodos e instrumentos pedagógicos contrarios ar la igualdad social o que mantengan una condición subalterna de las mujeres. Asi mismo, la Convención sobre Todas las Formas de Discriminación contra la Mujer (la cual fue ratificada en Costa Rica en el año 1984), en su artículo 10 , promulga la climinación de todo conceptoestereoiipado de los papeles masculinos y femeninos en todos los niveles y en todas las formas de enseñanza.

Por otro lado, el Código de la Niñez y la Adolescencia dictado por el Patronato Nacional de la Infancia (PANI) en 1998 plantea en el artículo 68, capítulo $V$, “ (...) la prohibición de promover o practicar en los centros educativos, todo tipo de discriminación por género, edad, raza u origen étnico o nacional, condición sociocconómica o cualquier otra que viole la dignidad humana" (p. 6). Asi tambićn, la Política Curricular hacia el Siglo XXI (MEP, 1994-1998), plantea la eliminación en el sistema general de la educación en particular, de los elementos que generen discriminación por razones de género o cualquier otra naluraleza.

Sin embargo, es pertinente señalar que a pesar de la normativa legal que existe en Costa Rica, en relación con el tema de sexismo según la percepción de los niños y las niñas, las investigaciones que existen, especialmente en el ámbito preescolar son escasas. Por ello se considera relevante la realización de la presente investigación con el fin de brindar un aporte significativo en este campo de estudio.

El sistema educativo debe garantizar la igualdad de oportunidades, esto involucra desde luego una equidad de sexos, que permita a la sociedad una adecuada distribución del poder y de la riqueza. Esto representa una de las finalidades que debe tener la educación, de esta manera se neutraliza el desartollo y la 
producción de violencia entre sexos. En este sentido, es importante recalcar que desde Épocas anteriores y hasta el momento, se continúa observando signos de discriminación, principalmente hacia el sexo femenino.

Ante este panorama, es necesario conocer más acerca del tema del sexismo, para brindar alternativas que estén dirigidas a evitar el sexismo que pueda estar presente en las relaciones de aula. De esta manera, se puede lograr un trato igualitario tanto para hombres como para las mujeres que comiencen desde temprana edad y que conlleve a obtener una sociedad libre de prejuicios y discriminaciones de este origen.

Con respecto a lo anterior, se realizó una investigación (Corrales, Delgado, Herrera, Percira, Sancho y Zamora, 2004), de la cual se aporta en este artículo una parte de los datos obtenidos y que tenía como finalidad analizar las concepciones de los niños y las niñas de 5 a 6 años acerca de los roles femeninos así como masculinos que permiten derivar si el sexismo es una realidad presente en el aula preescolar. Por lo tanto, partiendo de ahí, proporcionar mayores conocimientos acerca del tema que permitan conocer poco a poco, signos que puedan connotar discriminación de un determinado sexo.

Es pertinente señalar que la sociedad se encuentra cargada de sexismo, por lo tanto, aunque se han ido dando muestras de cambio, los roles sexuales establecidos y las costumbres existentes se encuentran muy profundos, por lo que se deben hacer esfuerzos sistemáticos para combatirlo. Sin embargo, esta transformación puede surgir de modelos educativos libres de sexismo, ya que se pueden fomentar las actitudes no discriminatorias para ir logrando personas conscientes de la necesidad de crear una sociedad cuyas prácticas sociales sean más igualitarias, para que tanto hombres como mujeres, ocupen el lugar que merecen dentro de una sociedad que es forjada por ambos sexos y que debe reflejar un trato justo $\mathrm{e}$ igualitario entre los individuos.

Por ser la escuela la primera institución formal por la que pasa lodo ser humano, se le recarga la obligación de instruir al mismo en su proceso de desarrollo. Hoy día y desde mucho tiempo atrás, se ha venido dando una discriminación de sexos masculino y femenino. Día a día la sociedad va cambiando y los roles de mujeres y hombres también, sin embargo el valor que se le da a la mujer se ha mantenido estático y a un nivel inferiorque el del género masculino. Sólo buscando alternativas se podrá ir disminuyendo el alcance de esta situación. Es por ello que se deriva la importancia de buscar la equidad de los mismos en defensa de la discriminación de la mujer prioritariamente. Según el Estado de la Nación (2000): 
"La aspiración central del ser humano es la existencia de oportunidades crecientes para el acceso de hombres y mujeres a una educación que les permitan mejorar sus condiciones de vida, que apoyen los procesos de movilidad y ascenso social y reduzcan las brechas entre los diferentes grupos de población, sectores y áreas geográficas" (p. 269).

Retomando lo planteado es importante reconocer que la institución educaltiva es vista como uno de los medios que le ayuda al ser humano a mejorar sus condiciones de vida; entendiendo estas en el amplio sentido de la palabra, es decir que haya un crecimiento económico, cultural, social y sobre todo moral que le permita crecer. En una sociedad donde por mucho tiempo se le ha restado importancia al sexo femenino, es necesario hacer algo para cambiar esta situación y es por ello que es importante trabajar temas que ayuden a variar la forma de pensar de los seres humanos en la escuela y desde el nivel preescolar.

En este sentido hay planteamientos teóricos que indican que se ha de hacer visible lo invisible y una forma de lograrlo es poder profundizar en este tipo de temáticas y hacer evidente la existencia o no de manifestaciones sexistas en el ámbito educativo entre otros. Si se analiza con detenimiento el valor tan importante que se le da a la educación se puede entonces comprender que es la herramienta ideal para desaurollar temas que atañen a toda la sociedad, y que de una u otra manera generan impacto social.

Es evidente por lo tanto, que el sexismo se muestra en la sociedad de divef sas maneras y que a pesar de los esfuerzos que muchas personas realizan para ir erradicándolo sigue presente aún en la actualidad. Es muy importante tomar en cuenta lo que expone Lagarde (1999):

(...) el sexismo es generado socialmente porque parte del orden, de los mecanismos de funcionamiento, de las estructuras y las relaciones sociales recrean formas de dominación basadas en el sexo de las personas y en lo que las personas hacen con su sexualidad (p. 67).

Precisamente, éstas manifestaciones de sexismo que se dan en la sociedad, son tan comunes que la mayoria de las veces se presentan de forma espontánea, es decir, la persona no considera que está siendo sexista, porque es así como ha aprendido a comportarse desde que es niño o niña. En este sentido, las actitudes de los y las infantes denotan mucho la conducta sexista, ya que desde que nacen están expuestos y expuestas a eliquetas y estereotipos asignados por sus progenitores y a su vez asignados a éstos (as). De esta forma se puede creer que la sociedad es la responsable de que estas conductas sexistas, se sigan manteniendo. 
Por otra parte, se considera que la familia es la base de la socicdad, por lo tanto ésta como seno formador y primera instancia socializadora del ser humano poscc una responsabilidad directa de los valores y creencias quc transmite a sus hijos e hijas. Por eso, cuando el padre, la madre u otra persona cn la fiamilia poscc creencias sexistas, éstas sc transmiten a los niños y las niñas continuando la reproducción de las mismas de gencración en generación, hasta que las mismas personas hagan conciencia de que es neccsario romper con cstc ciclo de reproducción. Haciendo alusión a un ejemplo muy común de manifestaciones sexistas dentro de la familia se puedc mencionar cl siguientc:

Mi papá es el jefe de la familia. Él trabaja para alimentamos, vestirnos y cducamos, cnviándonos a la cscucla. Él cs muy bueno. Mi mamá cs la que se cncarga del cuidado de la casa. Ella prepara las comidas, lava, plancha y arregla la ropa. Ella también es buena (Diaz y Díaz, citados por Gonzílcz, 1990, pp. 89-90).

Expresioncs como la antcrior son muy comuncs, pues la forma de vida del costarricense se ha centrado desde muchos años en el patriarcado como ya sc ha mencionado, y no cs fácil crradicarlo. En este sentido, es importantc anotar la división que se hace en la cita anterior de las funciones del papá y las de la mamá, ya que son las más comuncs en nucstra socicdad y muchas veces cstín basadas en estercotipos sexualcs, que son los que marcan el scxismo.

Por lo tanto, el scxismo es un clemento presente en la socicdad que sc marca en las condiciones de vida que existen para los hombres y para las mujeres, las cuales son difcrentes como consecuencia de la desigualdad sexual. Así mismo, este sc pucde manifestar de diversas maneras y en diferentes nivelcs. que van de acucrdo a las conductas, creencias y forma de vida de las personas que conforman la socicdad.

\section{Metudología}

La investigación fue de caráctcr cxploratorio. De acuerdo con el plantcamiento de Barrantes (2000) se definió como "(...) una investigación que sc realiza para obtencr un primer conocimiento de una situación (...) por eso se dice que tiene un caráctcr provisional" (p. 64). Se consideró que éste cs el tipo de estudio porque no existen otras investigaciones que aborden el tema desde la perspectiva que se hizo en ésta, aunque si hay algunos que lo hacen desdc olias ópticas. 


\section{Definición de términos}

Sexismo: "(... ) se denomina sexismo a todos aquellos procesos que limitan el desarrollo de las potencialidades esenciales debido al sexo de cada persona y por ende de su grupo de iguales (...), cl sexismo es la discriminación de individuos $\mathrm{c}$ individuas relacionada con la pertenencia a un sexo determinado". (Gonzálcz, 1990, p. 11).

Roles o papeles sexuales: cstán relacionados con cl comportamiento de los scres humanos de acuerdo al sexo al que pertenecen, a saber: femenino o masculino, respondiendo esto a un tipo de sociedad detcrminada. Éstos son fortalecidos tanto en la familia, como en la cscucla y de cse modo se incorporan a la conducla de cada persona.

\section{Participantes}

Docentes: para efectos de la investigación (Corrales et al. 2004), sc contó con la participación de un total de 20 docentes, que laboraban en los jardines infantiles públicos en los cuales se reali zó cl cstudio. Las instituciones participantes fucron: Pedro Murillo Pércz, Margarita Esquivel, Dario Flores Hernández, José María Castro Madriz y Eulogia Ruiz Ruiz, y se escogicron bajo los critcrios de acceso y disponibilidad de recursos.

Cabe destacar, que de cada institución se escogicron cuatro docentes por parte de las investigadoras y todas fucron docentes que laboraban en Educación Precscolar y algunas específicamente en el ciclo de Transición.

Niños y' niñas: para cl citado cstudio, sc contó con la participación de 60 cstudiantes de 5 a 6 años de cdad, que asistian al jardín infantil público. Los niños $(30)$ y las niñas (30) fucron scleccionados al azar.

No obstante, para cfecto del presentc artículo y por razones de espacio, sc enfatizará únicamente en la información aportada por niños y niñas precscolares y relacionadas específicamente con sexismo.

\section{Instrumentos}

Para la recopilación de la información se contó con tres tipos de instrumentos: la cutrevista dirigida a los niños y niñas participantes, cl cuestionario propucsto a las docentes involucradas dentro de la investigación y la lista de cotejo con la finalidad de conocer las manifestaciones de sexismo desarrolladas dentro del aula precscolar. 


\section{Aspectos éticos}

El estudio contó con todos los permisos correspondientes tanto por parte de administradores (as) como de las (os) docentes de las instituciones educativas involucradas en el cstudio, así como de los padres, madres de familia y de los niños y niñas participantes.

Las instituciones y los grupos involucrados contaron con la devolución de los resultados, una vez finalizado cl cstudio.

\section{Resultados}

Se destacan a continuación los aspectos más relevantes reficridos al sexismo en cl aula precscolar costarricense, bajo el entendido de que los resultados no son generalizables, sino que se circunscriben a las instituciones en las cuales se llevó a cabo el cstudio, no obstante, son un indicador de lo que sucede en aulas precscolares del sistema educalivo costarricense.

Los gráficos que se incluyen permiten al lector o lectora, visualizar de una mancra más global los datos obtenidos en este cstudio. Han sido claborados tomando en cuenta los aportes de las listas de cotcjo y considerando al tolal de 30 niños y 30 niñas. Es importante mencionar que el propósito fundamental de éstos es el de poder observar a nivel global las manifestaciones de sexismo en los y las preescolares, por cllo se considera la totalidad de los participantes y no son contabilizadas tampoco las opiniones negativas acerca de cada uno de los aspectos valorados.

Para cl resto de la información mediante cuadros se hace la diferenciación por sexo, lo cual permitirá una comprensión de las existencia o no de manifestaciones sexistas en aulas preescolares coslarricenses.

\section{Cuadro 1}

Juguetes preferidos por nin̄as y nin̄os, según categoría de respuesta

\begin{tabular}{|c|c|c|}
\hline Calcgorias de respuestas & Niñas $n=30$ & Niños $n=30$ \\
\hline & $\%$ & $\%$ \\
\hline
\end{tabular}

Juguctes asociados

Socialmente al género.

("bola", "muñecas",

27

90

24

80

"carros").

(Contimúa cuadro) 


\begin{tabular}{|l|cc|c|}
\hline Categorías de respucstas & \multicolumn{2}{|c|}{ Niñas $n=30$} & \multicolumn{2}{|c|}{ Niños $n=30$} \\
& $f$ & $\%$ & \multicolumn{2}{c|}{$f$} & $\%$ \\
\hline
\end{tabular}

Juguetes que están de

Moda

("Blay blade", "Hulk").

13

36

20

Juguctes electrónicos

("play station", "computadora") 2

El cuadro número uno hace referencia a los juguetes preferidos por niños y niñas, los cuales fueron ubicados por catcgoría de respuesta, es interesante señalar que tanto niñas (27) como niños (24) en mayor medida mencionan juguetes que socialmente han sido asociados al genero, por ejemplo, muñecas, carros, entre otros.

Algunos de los argumentos que dan las niñas al cscoger este tipo de juguetes son: "tracn muchas cosas, cama, hijo, cocina", "puedo jugar casita", "son bonitas", "juego de mamí, de lía, de escuclita y me divierto mucho", "son !indas y tienen el pelo largo", "tienen pelo largo, vestido largo, zapatos, hija, tren, prensitas para peinar".

Por otra parte, los niños dan argumentos tales como los siguientes: "sirve para hacer batallas", "porque cs rápido", "le pega a la perra cuando me moleslil", "me gusta manejar", "es muy' poderoso", "es fuerte".

Aparecen lambien en los datos, aunque en menor frecuencia, juguetes que cstán de moda o se intensifica su utilización por medio de la propaganda de los medios de comunicación y son justamente los niños los que suelen mencionarlos con mayor frecuencia.

Es interesante mencionar que los juguetes preferidos por las niñas y los niños se constituyen en un reflejo de los estereotipos de papeles sexuales que predominan culturalmentc (Hurlock, 1988). 


\section{Cuadro 2}

Categorias de profesiones u oficios por los que optarian niñas y niños cuando sean grandes, según frecuencia y porcentaje

\begin{tabular}{|c|c|c|c|c|}
\hline \multirow[t]{2}{*}{ Calcgorias de respucstas } & \multicolumn{2}{|c|}{ Niñas $n=.30$} & \multicolumn{2}{|c|}{ Niños $n=30$} \\
\hline & & $\%$ & $f$ & $\%$ \\
\hline $\begin{array}{l}\text { Prolesiones asignadas } \\
\text { socialmente por géncru. } \\
\text { (vetcrinario, macstro, policia) }\end{array}$ & 21 & 70 & 15 & 50 \\
\hline $\begin{array}{l}\text { Oficios asignacios socialmentc } \\
\text { por géncro (taxista, chofer, } \\
\text { vendedor). }\end{array}$ & 7 & 23 & 12 & 40 \\
\hline $\begin{array}{l}\text { Profesiones artísticas } \\
\text { (cantante). }\end{array}$ & 2 & 7 & 1 & 3 \\
\hline $\begin{array}{l}\text { Personajes fantásticos } \\
\text { (Hulk, Batman). }\end{array}$ & - & - & 2 & 7 \\
\hline
\end{tabular}

En cl cuadro númcro dos, ante la pregunta qué tc gustaría scr cuando seas grande, niñas y niños mencionan prioritariamente profesiones $u$ oficios que socialmente han sido asignados per géncro. Para la calcgoría de profesiones asignadas por género niñas (21) y niños (15) optan por alguna profesión que habitualmentc ha caractcrizado a hombres o mujercs. De igual mancra, cligen oficios asignados socialmentc por género ubicándose niñas (7) y niños (12) de esta calegoría.

Asimismo, las niñas (2) y un niño (1) descarían tener profesiones artisticas. Por otra parte, hay niños (2) que cuando grandes manifiestan que dcscan ser personajẹs fantásticos. 
Cuadro 3

Profesiones u oficios en que deben trabajar los papás de acuerdo a la opinión de niñas y niños ubicados, según frecuencia y porcentaje

\begin{tabular}{|c|c|c|c|c|}
\hline \multirow[t]{2}{*}{ Catcgorias de respucstas } & \multicolumn{2}{|c|}{ Niñas $n=30$} & \multicolumn{2}{|c|}{ Niños $n=30$} \\
\hline & & $\%$ & f & $\%$ \\
\hline $\begin{array}{l}\text { Oficios asignados socialmente } \\
\text { por género (vendedor, chofer). }\end{array}$ & 17 & 57 & 15 & 50 \\
\hline $\begin{array}{l}\text { Profesiones asignadas } \\
\text { socialmente por género } \\
\text { (veterinario, policía). }\end{array}$ & 7 & 23 & 12 & 40 \\
\hline $\begin{array}{l}\text { Profesiones y oficios } \\
\text { aceptados socialmente para } \\
\text { ambos géncros (profesor, doctor). }\end{array}$ & 6 & 20 & 3 & 10 \\
\hline
\end{tabular}

El cuadro número tres, indica por catcgorias, las profesiones $\mathrm{u}$ oficios $\mathrm{en}$ las cuales deben trabajar los papás scgún la opinión de niñas y niños. Es importante señalar que tanto niñas (17) como niños (15) indican con mayor frecuencia oficios que socialmente son asignados por género para hombres. De igual manera un número significativo de niñas (7) y de niños (12) mencionan que los papás deben trabajar en profesiones asignadas socialmente para hombres.

Con una menor frecuencia se mencionan también profesiones u oficios aceptados socialmentc para ambos géneros, talcs como ser doctor, profesor, trabajos de oficina, entre otros. Es así como se refleja en las respucstas aportadas por las y los infantes conceptos estercotipados acerca de los trabajos que realizan las personas adultas, donde se asocian las profesiones $u$ oficios de los hombres con aspectos de fuerza, poder, valentia e incluso inteligencia, (Castro, 1999). 


\section{Cuadro 4}

Profesiones u oficios en que deben trabajar las mamás de acuerdo a la opinión de niñas y nin̄os, según frecuencia y porcentaje

\begin{tabular}{|c|c|c|c|c|}
\hline \multirow[t]{2}{*}{ Catcgoriáas de respucstas } & \multicolumn{2}{|c|}{ Niñas $n=30$} & \multicolumn{2}{|c|}{ Niños $n=30$} \\
\hline & f & $\%$ & $\mathrm{f}$ & $\%$ \\
\hline Labores en la propia casa. & 10 & 53 & 14 & 47 \\
\hline $\begin{array}{l}\text { Oficios domćsticos fucra de la } \\
\text { casa (conscrje, cocinando). }\end{array}$ & 4 & 13 & 8 & 26 \\
\hline $\begin{array}{l}\text { Profesiones asignadas } \\
\text { socialmente por género } \\
\text { (enfermera, costurera). }\end{array}$ & 5 & 17 & 0 & 20 \\
\hline $\begin{array}{l}\text { Profesiones y oficios } \\
\text { aceptados socialmente para } \\
\text { ambos géncros (profesora, m }\end{array}$ & 5 & 17 & $\underline{2}$ & 7 \\
\hline
\end{tabular}

El cuadro número cuatro, scñala las profesiones u oficios en que deben trabajar las mamás según las opiniones de las niñas y los niños. En éste, sc indica que con regularidad las niñas (16) consideran que las mamás deben trabajar en labores propias de la casa, asimismo, los niños (14) scñalan con mayor frecuencia respucstas que se ubican en esa misma catcgoría.

Los oficios domésticos fucra de la casa sc ubican como scgunda catcgoria, ya que las niñas (4) y los niños (8) mencionaron respucstas tales como que las mamás deben trabajar cocinando, lavando o limpiando en otras casas. Luego se encuentran con menores frecuencias las catcgorias de profesiones que socialmente son asignadas para las mujeres y las que son aceptadas socialmente para ambos géncros.

Es interesante scñalar que las respucstas de las niñas y los niños indican que existe con mayor frecuencia condiciones estereotipadas que dividen los trabajos de acucrdo al géncro. 


\section{Cuadro 5}

Actividades que hacen las niñas en la casa de acuerdo con la opinión de niñas y niños ubicadas por categoria de respuesta, según frecuencia y porcentaje

\begin{tabular}{|c|c|c|c|c|}
\hline \multirow[t]{2}{*}{ Categorías de respuestals } & \multicolumn{2}{|c|}{ Niñas $n=30$} & \multicolumn{2}{|c|}{ Niños $n=30$} \\
\hline & & $\%$ & & $\%$ \\
\hline $\begin{array}{l}\text { Participar en juegos asociados } \\
\text { socialmente al género. }\end{array}$ & 17 & 57 & 18 & 61 \\
\hline $\begin{array}{l}\text { Ayudar en los quehaceres de la } \\
\text { calsia. }\end{array}$ & 9 & 30 & 9 & 30 \\
\hline Estudiar & 2 & 7 & 1 & 3 \\
\hline Usar juegos electrónicos & 1 & 3 & 1 & 3 \\
\hline Ver televisión & 1 & 3 & 1 & 3 \\
\hline
\end{tabular}

En el cuadio cinco ante la pregunta qué actividades hacen las niñas en la casa, niñas y niños mencionan en forma prioritaria criterios relacionados con la categoría que se refiere a participar en juegos asociados socialmente al género. Así las niñas (17) y los niños (18) mencionan como actividades a realizar por las niñas algunas como las siguientes: jugar con muñecas, barbies, de casita, entre otras.

Asimismo, en una segunda categoría en cuanto a frecuencia, niñas (9) y niños (9) señalan el hecho de que las niñas en la casa lo que hacen es ayudar en los quehaceres. Tambien mencionan otras actividades como el estudiar, usar juegos electrónicos y ver televisión, pero en menor frecuencia.

Los datos ponen de manifiesto que socialmente se marca una división entre géneros que ubica a la mujer como la encargada de hacer los oficios, cuidar del hogar y que este rol estereotipado es el que debe ser transmitido culturalmente (Castro, 1999). 


\section{Gráfico 1}

Opiniones de los y las preescolares acerca de si son las mujeres o los hombres quienes deberian encargarse de realizar las labores de crianza

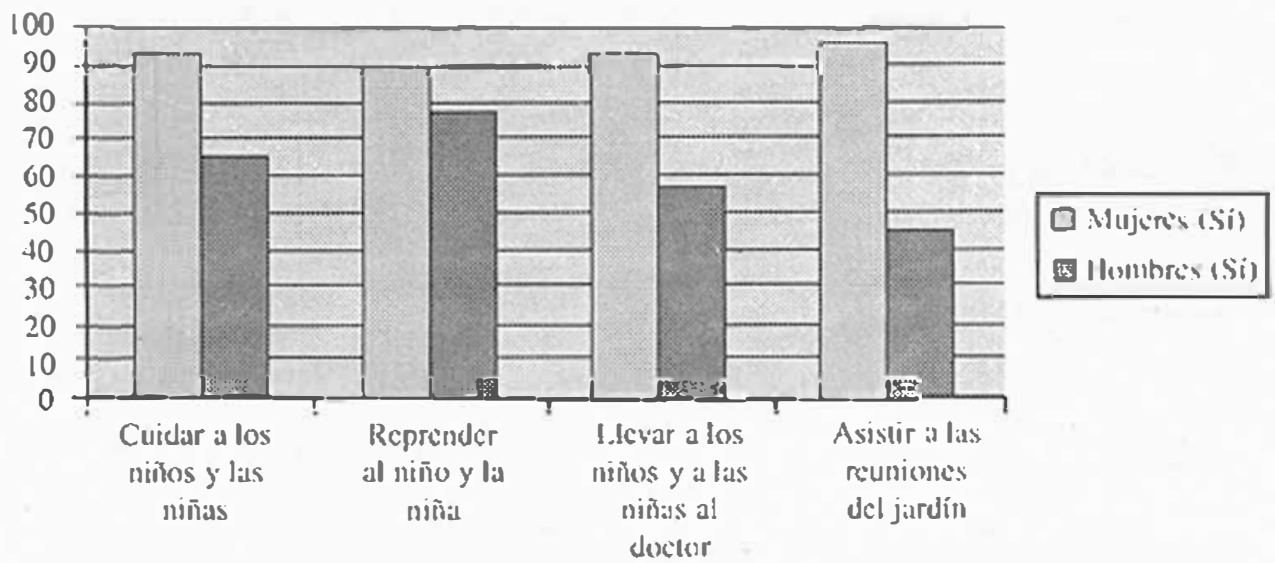

El gráfico I mucstra las opiniones de los y las precscolares acercal de si son las mujeres o los hombres quienes deberían encargarse de realizar diferentes labores relacionadas con la crianza de los hijos y las hijas. En términos gencralles los datos mucstran que son las mujeres las que mayoritariamente deberían encargarse de llevar a cabo las labores relacionadas con la crianza de los y las menores. No obstantc, tanto niños como niñas consideran que dichas labores podrian ser realizadas por varones, pero en menor escala. Fs por ello que se puede decir que los dalos coinciden con los plantcamientos teóricos en donde se priorizan cicrtas labores de crianza para cl sexo femenino, pero tambićn se reconoce la posibilidad de que cstas scan cjeculadas por varoncs.

Es notable ver que en aquellas actividades con responsabilidades específícas de mayor cuidado como lo son cl csludio y salud de los y las menores; los niños $y^{\prime}$ las niñas encuestados en su mayoria (.50 correspondientc a un 93\%) consideran que debe ser la mujer la que se encargue de llevar a cabo actividaldes como llevar a los niños y las niñas al doctor. También, se presental cl caso de responsabilidades como asistir a las reuniones del jardín, en esic aspecto tanto niños como niñas mayoritariamentc (57 cquivalentc a un $95 \%$ ) crecn que deben ser las mujeres las que se encarguen de dichal actividad. Sin cmbargo, 27 niños y niñas $(45 \%)$ crecn que los hombres también podrían lleval a cabo estc tipo de aclividades. 


\section{Gráfico 2}

\section{Opiniones de los y las preescolares acerca de sí son las mujeres o los hombres quienes deberían realizar los oficios domésticos en el hogar}

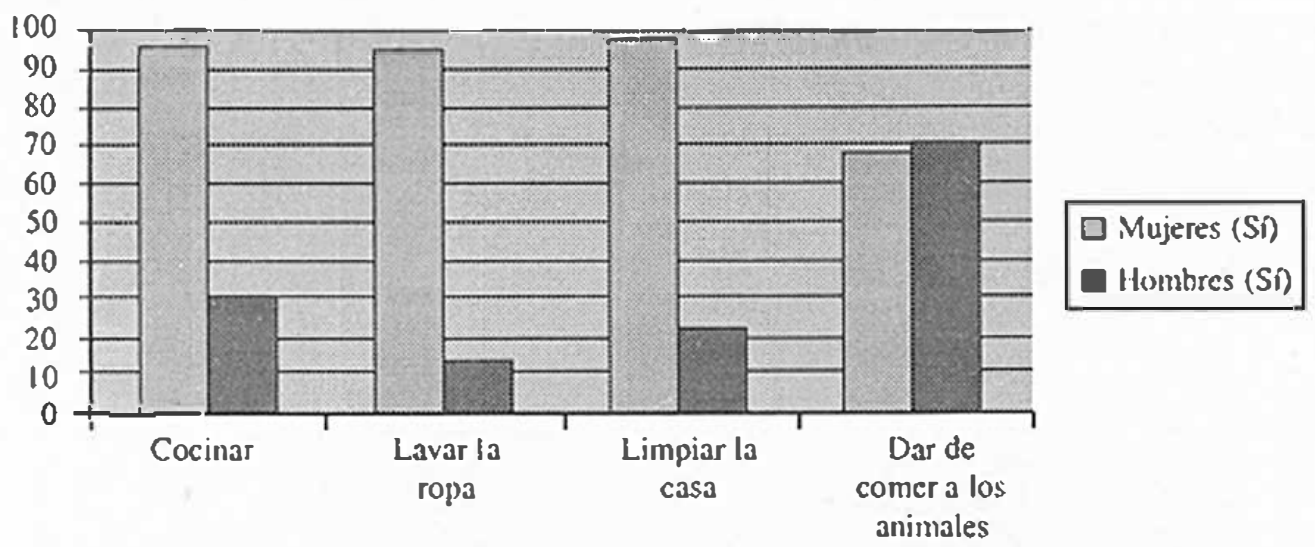

El gráfico 2 muestra las opiniones de los y las precscolares acerca de sí son las mujeres o los hombres quienes deberian encargarse de rcalizar los diferentes oficios domésticos en el hogar. Aquí se hace relcvantc mencionar a Rommetveit (1976), quien menciona que a los niños y las niñas cuando sc les cuestiona acerca de temas relacionados con el papel de las mujeres y hombres, parten de referencias implícitas y explícitas a la rutina cotidiana de la vida de su propio padre y madre. Es intcresante poder observar la coincidencia que sc da entre este planteamiento teórico y la realidad reflcjada por los y las participantes. Así por ejemplo, se puede mencionar el caso de oficios como lavar la ropa en el cual la mayoría de los niños y las niñas ( 57 correspondientc a un 9.5\%) consideran que deberían ser las mujeres quienes sc encargucn de esta labor; mientras que solo 8 niños y niñas (13\%) creen que también los hombres podrian realizarla. Además, limpiar la casa es una actividad que casi en su totalidad niños y niñas (.59 equivalente a $98 \%$ ) piensan que debería ser responsabilidad de las mujcres y solo 14 niños y niñas (23\%) consideran que los hombres tambićn podrían encargarse.

Es por ello que puede inferirse que los y las preescolares entrcvistados mayoritariamente, consideran que todos los oficios domésticos que se realizan en cl hogar deberian ser responsabilidad de la mujer principalmentc, respondiendo así al paradigma tradicional en donde los roles están específicamentc delimitados para cada sexo. 
No obstante, por estar viviendo en una etapa de cambio, se puede observar que empiezan a surgir vestigios de un cambio de paradigma, ya que en actividades como darle de comer a los animales tanto niños como niñas (40 correspondiente a un $67 \%$ ) consideran que las mujeres deberían de encargarse, sin embargo, 41 niños y niñas (68\%) parecen darse cuenta que los hombres podrían hacerlo también, siendo este el único de los oficios compartidos que se obtiene a partir de los datos obtenidos de las listas de cotejo.

\section{Gráfico 3}

Opiniones de niños y niñas accrca de quién se debería encargar de brindar el soporte económico al hogar

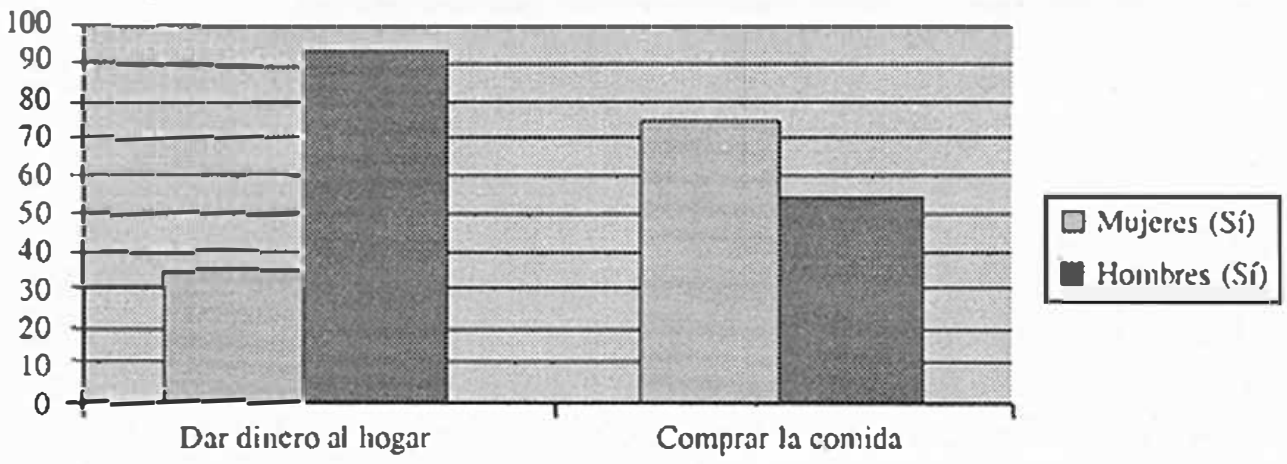

El gráfico 3 muestra las opiniones de los y las participantes acerca de sí son las mujeres o los varones quienes se deberían encargar de brindar el soporte económico al hogar. Debe mencionarse que los y las participantes, parten de su propia realidad para poder dar una respuesta. Es por ello, que la mayoría de los niños y las niñas entrevistados y entrevistadas (56 correspondiente a un 93\%) consideran que dar dinero al hogar debería ser una responsabilidad de los hombres, ya que esto probablemente es lo que refleja la dinámica familiar y cultural en la que se desarrolla. Cabe mencionar que dentro de esta realidad, los medios de comunicación son muy influyentes.

Asimismo, comprar la comida es una actividad que ubican dentro de las responsabilidades de las mujeres principalmente. Tanto niños como niñas en su mayoría (45 equivalente a $75 \%$ ) tienen la noción de que comprar la comida es únicamente el acto de ir al supermercado y hacer las compras, ya que de acuerdo a los datos de este gráfico pareciera que quienes aportan el dinero para la compra de la comida son los hombres. 


\section{Gráfico 4}

\section{Opiniones de los y las preescolares acerca de las actividades que pueden realizar hombres y / o mujeres}

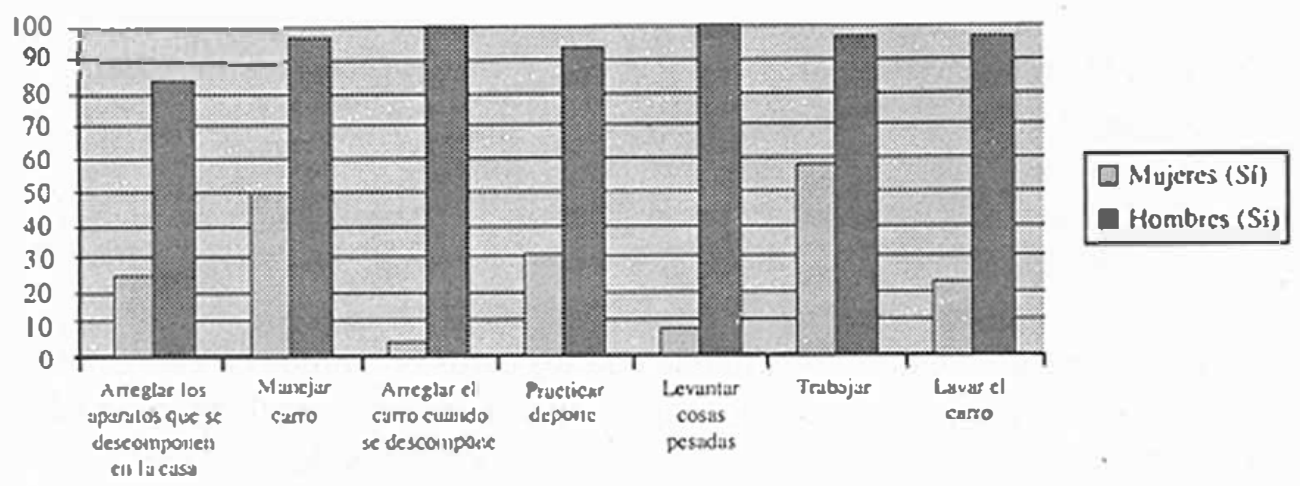

El gráfico 4 mucstra las opiniones de los y las precscolares accrca de algunas de las actividades que a su criterio pueden realizar hombres y / o mujeres. En términos gencrales los datos muestran que todas aquellas labores que requicran de una disciplina o entrenamiento más puntual y donde la fucrza física entra en juego son realizadas mayoritariamentc por los hombres, sin cmbargo, aceptan que las mujeres podrian realizar algunas de estas, pero en menor cscala.

En este sentido, podríamos empezar analizando la actividad de arreglar el carro cuando se descomponc. Tal y como se muestra en el gráfico los 60 niños y niñas correspondientes al $100 \%$ de la población encucslada consideran que este lipo de actividad es realizada por varones, mientras que únicamentc 2 niños y niñas $(3 \%)$ piensan que una mujer podría arreglar un carro.

La misma situación se presenta al cuestionar a los y las participantes en el estudio con respecto a quien deberia levantar las cosas pesadas. El $100 \%$ de ellos ( 60 niños y niñas) continúan reflcjando un pensamiento sexista, en donde expresan que son los hombres quienes deberian encargarse de levantar cosas pesadas. No obstante, un $8 \%$ de la población ( 5 niños y niñas) inician en un periodo de cambio de mancra que podrian considerar a las mujeres capaces de llevar a cabo aclividades como la mencionada.

Por su parte, aquellas actividades que se realizan por lo general fucra del hogar como practicar deportc y manejar el carro son actividades que los niños y las niñas asignan en su mayoria a los hombres. Sin embargo, admiten que son 
actividades que podrian ser realizadas por las mujeres, obviamente las últimas en menor escala.

Asimismo trabajar es una actividad que 59 niños y niñas (98\%) asignan como responsabilidad de los hombres. Al respecto Arenas, et al. (2000) señala que al hombre se le han asignado papeles de índole puiblica; por ejemplo

"(... ) la política, los negocios, la cultura, el trabajo remunerado, etc), porque el hombre no asume, comparte, ni desartolla los roles femeninos. Las niñas (y también los niños) son educadas desde pequeñas en esa dirección, para responder a las demandas y expectativas sociales sobre su género" (p. 12).

Este planteamiento es coincidente con los datos obtenidos en este estudio, pues aunque día a día sean más las mujeres que se incorporan al mercado labo. ral; los niños y las niñas parecen no darse cuenta, ya que de los 60 niños y niñas encuestados solo 35 menores (58\%) asumen que la mujer podría trabajar, entendiéndose por este trabajo todas las actividades que se realizan fuera del hogar y no son oficios domésticos.

\section{Consideraciones finales}

En términos generales y a manera de síntesis, puede indicarse que a partir de los datos aportados en el presente estudio, es posible señalar que existe sexismo en las aulas escolares en las cuales se realizó el estudio. De igual manera es posible señalar que niñas y niños preescolares asignan a la mujer una labor que la somete fundamentalmente a labores propias del hogar y del cuidado de los niños y las niñas. Aunque eventualmente también se visualiza por parte de los y las preescolares la posibilidad de que las mujeres puedan ejercer otro tipo de funciones. En este sentido, coincide lo encontrado con los planteamientos teóricos que señalan entre otros aspectos, que la mujer se ha visto reducida a un solo ambiente, es decir, el hogar; esto producto a las ideologías que se tiene en el sistema patriarcal, según el cual una mujer vale comolal si se dedica únicamente a atender a su pareja, sus hijos e hijas y desde luego su hogar; esto sin intentar realizarse en otras áreas que le permitan permanecer fuera de su casa.

Por otral parte, los datos recopilados indican que tanto niñas como niños, visualizan al hombre dentro de un estereotipo de papel sexual en el que se prioriza la fortaleza y la superioridad sobre la mujer, aunque cabe también destacar que se vislumbran opiniones que aceptan la participación de los hombres en labores que socialmente siempre han sido asignadas a mujeres. Esto es una muestra más 
de la coexistencia paradigmálica en relación con el género, aunque es aún muy fuerte la visión del hombre que plantea la mayoría de estudios y cuyos datos coinciden con los encontrados en la investigación cn aulas costarricenses, en los que se señala que es el que gencra ingresos económicos al hogar, mientras que la mujer es la que se encarga de velar porque éste este ordenado; por lo tanto el hombre es el que tiene derecho a cstar fucra del mismo.

Es intcresante señalar quc los datos de la investigación plantean la cxistencia de scxismo en las aulas preescolares, donde niñas y niños manifiestan una priorización del hombre sobre la mujer en rclación con las actividades 0 situaciones que puede vivir. No obstantc, $\mathrm{cn}$ algunas niñas y niños comicnzan a aflorar opinioncs de mayor apertura hacia el papel sexual de la mujer y del hombre, lo cual bien podría ser un reflcjo de cambio en los patroncs de crianza dc algunas familias.

Cabe recordar que las nociones de los y las preescolares tienen una cstrecha rclación con los aprendizajes en sus hogares, por lo cual ha de destacarsc también, que aunque en menor proporción se escuchan por parte de los y las participantes, opinioncs que dejan ver la apcrtura para quc hombres realicen funciones que habitualmente han sido asignadas a las mujercs y viceversa, cs posible entonces pensar que la institución educativa, al igual que la familia, podría cumplir un importantc papcl en la disminución del sexismo en el ámbito educativo.

En csic sentido, podría pensarse que emergen ya en cl ámbito preescolar, plantcamientos y opiniones que responden al nucvocstcreotipo de papeles scxuales, basado en la igualdad de la mujer. Razón por la cual, se fortalccc cl criterio de que la docente dcl nivel preescolar, podria favorccer ampliamentc cl afianzamiento de critcrios en las niñas y niños mediante los cuales se promueva la igualdad y el respcto, sin discriminación por género.

\section{Referencias}

Aguilar, L.; Barley, M.; Bertozzi,Y.; Calvo, Y.; Camac, E.; Camacho, L.; Escalantc, A.; Facio, A.; González, M.; Gutiérrez, E.; Quirós, E.; y Trcjos, M. (1995). ¿Feminismo en Cosıa Rica?. Testimonios, reflexiones; ensay'os. San José, Costa Rica: Mujeres.

Arenas, G.; Blanco, N.; Casłañcda, R.; Hernándč, G.; Jaramillo, C.; Moreno, E.; Oliveira, M.; Santos, M.A. y Simón, M.E. (2000). El harén pedagógico. Perspecriva de género en la organización escolar: Barcclona, España: Editorial GRAÓ. 
Ayales, 1.; Beniter, M.: Fevrier, S. y Ramirez, I. (s.f.) Género, commuicación y desarrollo sosienible: Aportes conceptuales y' merodológicos. San Josć. Costa Rica: IICA.

Barrantes. R. (2000). Investigación: un camino al conocimientu. un enfoque crantirativo y crualisativo. San José: Editorial de la Universidad Estatal a Distancia.

Castro, S. (1999). Socialización diferenciada por género. San José. Costa Rica: EDILOY.

Comalcs, L. ; Delgado, M. ; Herrcra, C.: Percira, 7. ; Sincho, J. y Zalnural, M. (2004). Sexismo en el aula preescolar: la perspectiva de los niñus y las miñas: de 5 a $\sigma$ años. Seminario de Graduación para Licenciatura no publicado. Heredia: Universidad Nacional.

Estado de !a Nación, informe No 7. (2000). San José, Costa Rica.

Ferro, C. y Quirós, A. (1998). Mujer, realidad religiosa y' communidad. (Iª cd.). San José, Costa Rica.

González, M. (1990). El sexismo en la educación: la discriminación coridiana. San José, Costa Rica: Editorial Universidad de Costa Rica.

Hurlock, E. (1988). Desarrollo del niño. (2ª ed. en español). México: Mc Graw-Hill.

Lagarde, M. (1999). Una mirada feminissa en el umbral del milenio. Heredia. Costa Rica: Instituto de Estudios de la Mujer.

Ministerio de Educación Püblica. (2000-2002). El sistema educativo nacional. Hacia una cultura de equidad de género. Plan de Acción Estratégico. San José, Costa Rica: MEP.

Rommetveit, R. (1976). Nonnas y roles sociales. Buenos Aires: Editorial PAIDOS. 